\title{
LA LUDOMOTRICIDAD EN EL PROCESO COGNITIVO DE LOS NIÑOS DE PRIMER GRADO DE EDUCACIÓN BÁSICA, CANTÓN LA LIBERTAD
}

\section{LUDOMOTRICITY IN THE COGNITIVE PROCESS OF FIRST GRADE CHILDREN IN BASIC EDUCATION, CANTON LA LIBERTAD}

\author{
Edwar Salazar Arango ${ }^{1}$ \\ edwarsalazar@hotmail.com / Esalazar@upse.edu.ec \\ Ana María Uribe Veintimilla ${ }^{1}$ \\ Mónica Dolores Tomalá Chavarría ${ }^{1}$ \\ ${ }^{1}$ Universidad Estatal Península de Santa Elena, Ecuador
}

\section{RESUMEN}

La educación integral, como pilar del sistema educativo ecuatoriano, establece potenciar el aprendizaje a través de experiencias vivenciales y significativas que se deben dar en ambientes adecuados y estimulantes; brindando un norte para justificar el presente artículo que constituye un análisis de la pedagogía lúdica y la psicomotricidad como una herramienta de mediación social - educativa, en que la relación psicosomática entre las acciones motoras, las respuestas psíquicas del aprendizaje, las emociones o sentimientos en una resonancia afectiva, crean el escenario para una combinación de componentes del desarrollo integral, enfocado a la educación formal o escolarizada. Por tal motivo, se direcciona un método de intervención didáctico a favor del aprendizaje, y cuya acción lúdica como herramienta pedagógica utiliza la exploración, descubrimiento, conocimiento y experimentación en busca de resultados en competencias corpóreas necesarias para las siguientes etapas del proceso evolutivo. Desde esta perspectiva se realiza un análisis descriptivo, revisión de fuentes teóricas, interpretación de datos obtenidos de los instrumentos investigativos de carácter empírico, aplicados a la población, objeto de estudio como son los niños de primer grado de educación general básica de las instituciones educativas fiscales del cantón La Libertad. El alcance investigativo, propuesta metodológica van enfocados a la identificación de diferentes estrategias, recursos educativos de carácter lúdico que, desde el juego formativo y direccionado con intenciones didácticas como acción participativa e incluyente, se adapta a las circunstancias particulares y cambiantes de los contextos escolarizados; cumpliendo con las recomendaciones o directrices del desarrollo integral infantil establecidas en el "Buen Vivir".

Palabras clave: Psicomotricidad - Desarrollo cognitivo - Educación inicial - Estrategias didácticas.

\section{ABSTRACT}

Integral education, as a pillar of the Ecuadorian educational system, establishes the promotion of learning through experiential and meaningful experiences which must be given in appropriate and stimulating environments, provides a north to justify the present article, which constitutes an analysis of playful pedagogy and psychomotricity as a tool of social - educational mediation, in which the psychosomatic relationship between motor actions, psychic responses of learning, emotions or feelings in an affective resonance; create the stage for a combination of components of integral development, focused on formal education or schooling; for that reason, a method of didactic intervention is favored in favor of learning and whose playful action as a pedagogical tool uses the exploration, discovery, knowledge and experimentation in search of results in corporeal competences necessary for the following stages of the evolutionary process. From this perspective, a descriptive analysis, revision of theoretical sources, interpretation of data obtained from empirical research instruments applied to the target population is carried out, such as first grade children of basic general education of fiscal educational institutions of Canton La Libertad. The research scope, methodological proposal is focused on the identification of different strategies, educational resources of a ludic character, that from the educational and directed game with didactic intentions as participatory and inclusive action, is adapted to the particular and changing circumstances of the schooling contexts; complying with the recommendations or guidelines of the integral development of children established in the "Buen Vivir". 
Keywords: Psychomotor - Cognitive development - Initial Education - Teaching Strategies.

Recibido: 9 de junio de 2017

Aceptado: 12 de Julio de 2017

Publicado: 31 de julio de 2017

\section{Introducción}

El juego, estimula e identifica cada uno de los elementos para el desarrollo psicomotriz de la población infantil; requiere de acciones metódicas, sistemáticas y didácticas que aporten al proceso de aprendizaje, el que se adapta a las circunstancias cambiantes del contexto formativo escolarizado o no; allí el cerebro goza de una participación ininterrumpida en su plasticidad, donde el docente o padre de familia pone en manifiesto su capacidad creadora para identificar como el desarrollo físico en su evolución kinestésica aporta de forma significativa al control motor en sus características gruesas y finas, habilidades cognitivas, comunicativas, socio afectivas.

El presente artículo, pretende en sus alcances descriptivos, bibliográficos y desde el trabajo de campo experiencial, plantear una variedad de estrategias, alternativas y acciones didácticas que, desde la pedagogía del juego, combinada con la psicomotricidad se establece un nuevo campo de acción, que se denomina ludomotricidad; la cual aporta al desarrollo del proceso cognitivo dentro de los derechos del Buen Vivir en la primera infancia. De manera que, los resultados expuestos sirvan de guía al profesional del área de las Ciencias de la Educación, de forma principal a los primeros niveles del proceso formativo (León, 2008) de las instituciones educativas fiscales del cantón La Libertad y a los padres de familia, encargados de cualificar y aportar al desarrollo integral del niño.

El juego es una actividad espontánea y placentera, que contribuye a la educación integral del niño, como elemento formador y desarrollador; se caracteriza como un medio de educación pedagógica, complejo. La acción lúdica y motriz del juego está dada en la formación de los hábitos motores, desarrollo y perfeccionamiento de cualidades vitales importantes, tales como: las destrezas físicas, intelectuales, morales y volitivas. De ahí su importancia y el interés en que se apliquen como elemento esencial dentro del sistema de formación en la Educación Inicial.

En los contextos escolarizados o ambientes familiares, se espera que las acciones formativas no se diseñen y apliquen de forma aislada al entorno del niño; pues, no son recetas que definen un solo proceso, ya que al hablar de formación integral infantil en las acciones de igualdad, equidad, cumplimiento de los estándares de calidad, se debe garantizar el desarrollo de las áreas motriz, cognitiva, afectivo-social y de lenguaje de los niños; éstas pueden surgir de la cotidianeidad en un contexto natural y espontáneo, imaginativo y creativo dedicando tiempo libre a actividades recreativas, lúdicas, de liderazgo, asociativas, deportivas y otras, para formar ciudadanos activos; tal como se lo establece en los lineamientos, políticas y objetivos del Plan Nacional del Buen Vivir (PNBV, 2013 - 2017).

\section{La psicomotricidad}

Los aportes de (Doidge, 2008) expresan que el cerebro está organizado "topográficamente", lo que significa que desde la sinapsis y mapa cerebral se van estructurando las acciones, tareas para el desarrollo de destrezas que realizadas de forma recurrente llevan a crear un hábito, una conducta que favorece las respuestas psicomotrices y éstas al aprendizaje.

Desde un aspecto formativo, la psicomotricidad es la que permite desarrollar, potenciar y conocer las habilidades, destrezas de la actividad corpórea; en ella, se puede comprender el cómo de la necesidad y acción del ser humano hacia el movimiento; la respuesta del proceso evolutivo que se organiza, estructura y consolida para obtener un adecuado dominio motriz, posibilitando en el niño diferenciación, autonomía, confianza y seguridad motriz en la relación directa y cotidiana del mundo natural, social, cultural.

La acción cerebral, identificada como la base para la acción motriz, desde la neuroplasticidad que tienen los niños en edad temprana, favorece para que se creen hábitos, conductas y patrones psicomotores que organizados en ambientes pedagógicos favorecen el aprendizaje. Allí, se establece la necesidad de una educación infantil.

El desarrollo evolutivo psicomotriz pasa por tres niveles principales: el primero la integración neuronal y activación del Sistema Nervioso Central (S.N.C) a favor de respuestas por 
estímulos, esto conlleva al segundo nivel o producto como una relación consecuente del niño con el medio o contexto y posterior o tercer nivel, a la materialización o instrumento de formación de la consciencia; tal como lo expone (González, 2015), quien toma a Da Fonseca (1988), entendiendo que las interacciones y relaciones de los procesos de la acción motora, requieren de voluntad, interés, emoción, expresión, afectividad y de elementos cognitivos, psíquicos en la búsqueda del pensamiento, necesarios en el aprendizaje.

\section{Objetivo de la psicomotricidad}

El desarrollo y evolución psicomotriz no es algo que simplemente se da en el niño, puesto que éste, según sus espacios y ambientes estimulantes va construyendo en el deseo inherente de explorar, descubrir; llevando a entender la meta y el objetivo de la psicomotricidad en conseguir el dominio y control del cuerpo pasando por estadios como la maduración de los reflejos, desarrollo de patrones básicos motores, habilidades y destrezas básicas motoras las cuales deben cumplir con las leyes y principios del desarrollo motor. Éstos determinan la organización del control motor que se puede evidenciar de forma céfalo - caudal: desde las extremidades superiores a las inferiores; próximo - distal: se maduran primero acciones motoras distales de la línea media del cuerpo; genérico específico: aparecen respuestas motoras más generales, gruesas y luego las específicas o finas. También, por su nivel de progresión que se evidencia por la maduración de los reflejos, seguido del tono y la estabilización muscular, la locomoción, la manipulación, el grafismo y la estructuración espacio - temporal: lateralidad, control postural, equilibrio (Justo M. E., 2014).

La intervención psicomotriz, aborda tres aspectos transversales como el terapéutico, educativo y re-educativo; enfocado a los diferentes aspectos del sistema psicomotriz, con sus respectivos ejes como el cuerpo (medio y fin formativo); producto de una relación inteligente entre el niño como el instrumento de formación y materialización de la consciencia. El eje del espacio y el tiempo como elemento de interacción de la acción motora que requiere de interés, motivación, voluntad (González, 2015).

\section{Corporeidad}

Se puede entender desde la frase "cuerpo vivo - cuerpo vivido" para lo cual se puede expresar del primer argumento que éste hace referencia a la respuesta física, natural del cuerpo y el segundo a los significados, emociones, sentimientos, acciones que se generan desde un cuerpo vivo. La corporeidad se establece desde la capacidad y desarrollo de elementos corpóreos que permiten al ser, poder conocer, sentir, aceptar, desarrollar y cuidar el cuerpo.

Las orientaciones educativas corpóreas, desde el conocer se refieren a la identificación de cada parte física en su estructura segmentada y total, sus funciones, control y respuestas ante estímulos externos e internos. El sentir hace referencia a comprender la relación de cada parte del cuerpo hacia la concepción del "yo", como una acción motora que da significancia y aporte a la identidad del ser; que conlleva al siguiente nivel progresivo de la corporeidad que se establece en la aceptación; comprensión de las diferencias, particularidades, limitantes y potencialidades del cuerpo en movimiento que puede llevar al ser a crear una actitud positiva corporal, que favorezca el poder desarrollar casi siempre de forma innovadora y creativa acciones motoras que pasan evolutivamente desde los reflejos, patrones, habilidades y destrezas básicas, capacidades condicionales. Se complementa la corporeidad con el cuidado del cuerpo hacia el entender y aplicar alternativas rutinarias para una adecuada salud física (Gallo, 2012)

\section{Ludomotricidad como método}

La lúdica como una actividad o ejercicio innato del ser humano que proporciona alegría, placer, gozo, satisfacción. Se dimensiona dentro de las necesidades del desarrollo humano a favor del tiempo libre, el juego y otras acciones que aportan a vivir y disfrutar cada acción de manera libre, espontanea e enriquecedora para el aprendizaje e integralidad; expuesto por (Yturralde, 2014).

De esta manera, se plantean los juegos como recurso metodológico y medio que ofrece situaciones lúdicas que permiten a niños y niñas asumir progresivamente la responsabilidad de sus aprendizajes. Los juegos permiten disfrutar, alegremente, y divertirse, evitando la discriminación; aportan a controlar su conducta, aseguran una relación armónica con sus compañeros y su medio social.

A través de la manifestación lúdica de la motricidad, se dinamiza el desarrollo de las capacidades motrices en un ambiente de libertad. Esta perspectiva de acción lúdica que proviene de los juegos de movimiento, además, de desempeñar un papel significativo en el 
desarrollo físico y psíquico del niño, constituye un excelente medio educativo que influye en la forma más diversa y compleja. El desarrollo de esta competencia implica la estimulación de habilidades y destrezas para resolver problemas de las áreas generales. Asimismo, incide en la creatividad para ajustarse a estructuras espacio - temporales, regulando las formas precisas de integración de su corporeidad en la ejecución de las acciones (Oseda, Mendivel, \& Zevallos, 2015).

La acción lúdica, además, de desempeñar un papel significativo en el desarrollo físico y psíquico del niño, constituye un excelente medio educativo que influye en la forma más diversa y compleja. El desarrollo de esta competencia implica la estimulación de habilidades y destrezas para resolver problemas de las áreas generales. Entre los aspectos que favorece el desarrollo psicomotriz desde la lúdica se tiene:

- Desarrolla y ejercita destrezas físicas, cognitivas, sociales; las cuales llevan al niño a ser un sujeto activo dentro del aprendizaje, para lograr su independencia en las distintas áreas del desarrollo psicomotriz.

- Se forman hábitos, conductas, patrones motores y psíquicos que en su esfera o dimensión espacio - temporal influyen en destrezas específicas en los conceptos de arriba - abajo, rápido - lento, derecha izquierda a favor de la sinapsis cerebral.

- Funciona como herramienta formativa para el ejercicio motor - cognitivo; el cual debe ser sistemático, vivencial y significativo para que sus efectos sean permanentes.

- Favorece el control del cuerpo y la mente a fin de producir respuestas motrices, cognitivas adecuadas ante las distintas situaciones que se le presentan, tanto en la vida escolarizada como en los diversos contextos donde convive.

- Apropiación de elementos, del esquema, la imagen y la conciencia corporal, equilibrio, tensión y relajación en actividades cinéticas y posturales, lateralidad, coordinación gruesa y fina.

- Destrezas para preguntar ¿qué debo hacer? ¿para qué lo haré?, ¿cómo y de qué forma lo puedo lograr?, ¿Qué resultados obtendré?

\section{Actividad física y psíquica}

Las relaciones motoras no están desligadas de las psíquicas, pues, a partir de las respuestas motoras el niño va construyendo diferentes esquemas mentales como inicio al desarrollo de la inteligencia, tal como lo expone (Piaget, 1971), el niño manipula y explora elementos en diferentes espacios, escenarios, generando una inteligencia práctica que con la significancia de valores afectivos puede llegar a interiorizar acciones reflexivas, para ser compartidas, relacionadas, comparadas con otras experiencias y personas en el tiempo y espacio, construyendo así las concordancias cognitivas que se dimensionan en un proceso organizado, continuo, progresivo de diferentes estructuras que se relacionan y entrelazan según las necesidades, intereses y estímulos.

Al continuar con los aportes de Piaget, para la edad de 2 a 7 años, en la que se encuentra incluida la población infantil escolar del primer grado de Educación Básica; se encuentra la etapa pre-operacional, en donde el niño desarrolla la capacidad de dar significado y representación a los símbolos, que aportan a la creación de esquemas mentales y preconceptos, finalizando con el estadio de la intuición e imitación de objetos, personas y escenarios que, enfocados de forma oportuna favorecen los aprendizajes (Gallego, 2010).

\section{Desarrollo de la capacidad cognitiva}

El ser humano por naturaleza pertenece a un entorno social, de una u otra forma siempre está en relación con alguien y en un contexto de interacción. Muchas son las circunstancias y ambientes que pueden aportar de forma significativa a los procesos cognitivos que se reflejan en el medio escolar; por lo cual, (Vargas \& Arán, 2014), proponen en su análisis investigativo sobre la cognición; algunos escenarios que favorecen el buen desarrollo de ésta competencia; empezando por describir que: los niños que en su estructura social tienen el acompañamiento de sus padres con una educación no autoritaria, participativa, reciben estímulos positivos para la innovación, la creatividad y el alcance de logros en la exploración y la autonomía personal.

(Escribano, 2004), expone que dentro de los principios del desarrollo cognitivo en el aprendizaje, se tiene como primer eslabón, que el niño debe ser su propio constructor de conocimiento, éste pasa por una fase de adaptación o acomodación en la medida que se transforman las percepciones cognitivas preconcebidas con las que requiere el mundo externo y las nuevas relaciones; esto permite entender que las situaciones, hechos, objetos pueden tener varias perspectivas de 
interpretación para relacionarse con el medio ambiente. El segundo principio, se basa en el descubrimiento por sí mismo, el niño es el actor de su aprendizaje, solo requiere de pautas y guías que faciliten entender la mayor cantidad de situaciones de exploración posibles; el tercer principio busca la individualización, entendiendo ésta, como la alternativa de crear experiencias a favor de las necesidades e intereses personales, sin olvidar las reglas y patrones sociales; que llevan a entender el cuarto principio que se basa en la interacción, en ella el niño debe poder ubicar una posición, una opinión y defenderla con criterio lógico.

\section{Materiales y métodos}

El tipo de investigación propuesto se basa en el enfoque cualitativo; se revisa, interpreta y valida los referentes conceptuales en la realidad cotidiana de la población, objeto de estudio para así identificar cómo el desarrollo psicomotriz puede influir significativamente en los procesos cognitivos de los estudiantes de primer grado de educación básica en las Instituciones Educativas del cantón La Libertad.

Por lo cual, la investigación, responde en su factibilidad a la necesidad de elaborar instrumentos como encuestas y fichas de observación que, al ser aplicadas, permiten identificar las percepciones de los docentes, y las acciones, comportamientos de los niños en su contexto real; que interpretadas y analizadas de forma correcta permiten describir alternativas de intervención, solución a la situación problémica y objeto de estudio.

Para este estudio, se consideró la población de las instituciones educativas del cantón La Libertad del área urbana que cuentan dentro de su oferta académica con el grado primero de Educación General Básica (E.G.B), de carácter fiscal o público; para un total de 28 instituciones; encontrándose con 54 docentes que imparten clases y 1890 estudiantes del nivel o grado en mención, para lo cual se aplica una muestra al número de niños, con probabilidad a favor del $3 \%$, probabilidad en contra del $1 \%$ y nivel de confianza del $100 \%$, obteniendo como resultado de muestra 47 estudiantes que son observados en su ambiente natural; por medio de una ficha, previamente, estructurada en relación a sus respuestas psicomotoras relacionadas a los procesos cognitivos.

\section{Resultados}

Los docentes identifican tener acceso a diferentes recursos para favorecer el desarrollo psicomotriz y al proceso cognitivo de los estudiantes; sin embargo, se evidencia poca aplicación de técnicas de aprendizaje que se relacionen en situaciones prácticas. Figura 1.

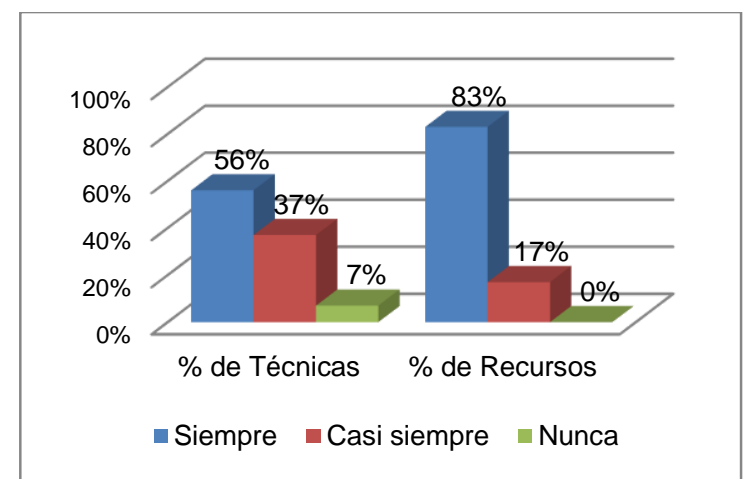

Figura 1. Uso de técnicas y recursos por parte del docente en situaciones prácticas

En las planificaciones docentes se aprecia que éstos, toman en cuenta "siempre" y "casi siempre" el nivel de desarrollo motriz de los niños y en esas mismas acciones, el proceso cognitivo no presenta las mismas referencias estadísticas, inclusive, docentes que nunca tienen relacionadas las dos variables (Figura 2).

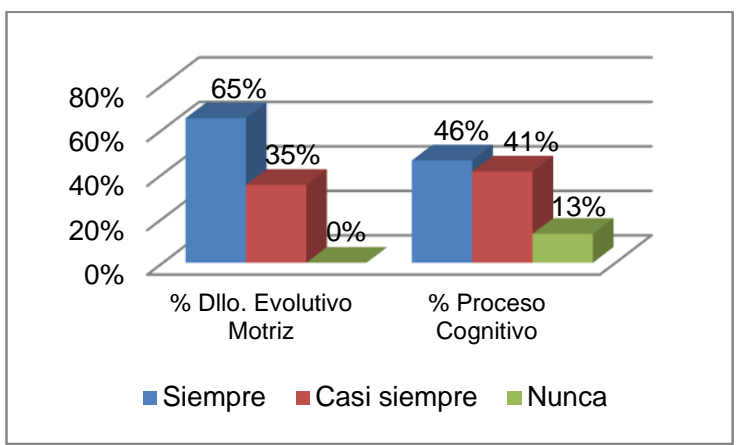

Figura 2. Relación del desarrollo motriz con el proceso cognitivo en la planificación escolar

Se puede inferir de la observación a los niños que hay una tendencia positiva de respuestas psico-motoras, la mayoría por el desarrollo evolutivo normal de los mismos; sin embargo, el que hayan respuestas de "algunas veces" y "nunca" amerita disponer de alternativas que en los procesos de enseñanza - aprendizaje escolar aporten a cualificar las competencias en los niños. 
Tabla 1. Respuestas psico-motoras en los niños

\begin{tabular}{|c|c|c|c|c|}
\hline INDICADOR & SIEMPRE & $\begin{array}{l}\text { ALGUNAS } \\
\text { VECES }\end{array}$ & NUNCA & TOTAL \\
\hline El niño se adapta en relación a situaciones motrices y cognitivas & 32 & 10 & 5 & 47 \\
\hline El niño da respuestas creativas e innovadoras & 28 & 12 & 7 & 47 \\
\hline Se identifican patrones y conductas motoras y psíquicas & 20 & 27 & 0 & 47 \\
\hline El niño expresa esquemas mentales y preconceptos & 38 & 9 & 0 & 47 \\
\hline El niño imita personas, animales y objetos & 40 & 7 & 0 & 47 \\
\hline
\end{tabular}

\section{Discusión de resultados}

La relación de las variables dimensionadas en los niños de primer grado de educación básica en donde se evidencia la importancia de aportar al desarrollo evolutivo natural de los niños con alternativas psicomotoras enfocadas a procesos cognitivos de enseñanza aprendizaje, se convierte en un espacio idóneo para adquirir destrezas que se sustentan en la plasticidad cerebral, flexibilidad cognitiva y la memoria (Owens, 2008).

Se puede deducir, entonces, que la psicomotricidad en su didáctica formativa ayuda a crear hábitos, conductas específicas a favor de la cognición; allí el niño interpreta el hacer motor con las ideas y preconceptos mentales que permiten en secuencia: plasmar sensaciones, pensamientos, conocimientos (Fernández, 2005).

Los conceptos expuestos a favor del desarrollo psicomotor en niños con edad preescolar proponen que; éste sea capaz de controlar su cuerpo a fin de producir respuestas motrices adecuadas ante las distintas situaciones que se le presentan, tanto en la vida escolarizada como en los diversos contextos donde convive.

La apropiación de elementos, del esquema, imagen y conciencia corporal, equilibrio, lateralidad, respiración, tensión y relajación muscular, orientación espacio - temporal, ritmo en diferentes actividades cinéticas y posturales se deben dar de forma progresiva al desarrollo evolutivo del niño.

Buscar y motivar que los niños realicen y contesten preguntas como: ¿Qué debo hacer? ¿Para qué lo haré?, ¿Cómo y de qué forma lo puedo lograr?, ¿Qué resultados obtendré? Para que los resultados y respuestas motoras sean de sus propias experiencias y den significado para la vida. Sustentado en la plasticidad cerebral, flexibilidad cognitiva, motivaciones intrínsecas puras de los niños en sus etapas evolutivas de desarrollo y crecimiento motriz, (Owens, 2008).
El autor hace referencia a que el niño puede dominar en su pasar formativo de la etapa preescolar un $80 \%$ de todas sus funciones evolutivas. Algunas características de ésta, con relación a la motricidad son:

- Tres años: Sube y baja las escaleras sin ayuda. Camina sin mirarse los pies al ritmo de la música; anda de puntillas, monta en triciclo, utiliza el cuchillo para extender. También explora, desmantela y desarma juguetes, toca instrumentos musicales sencillos.

- Cuatro años: Sube y baja las escaleras alternando los pies, salta sobre objetos en un solo pie, alternado y ambos. Puede copiar grupos de letras.

- Cinco años: Buen control motor y conocimiento de su cuerpo. Colorea sin salirse de las líneas y sus dibujos son más fáciles de reconocer. Se viste sin ayuda. Lateralidad manual bien establecida.

Los contenidos de formación psicomotor a favor de la cognición en sus escenarios globales, que se pueden perfeccionar a través de la lúdica y ambientes pedagógicos son:

Conocimiento del cuerpo en su escenario global y segmentario.

Adquisición de habilidades motrices básicas, aceptando y valorando a los compañeros y su propia participación en actividades colectivas.

Atención en diferentes señales comunicativas: gestos, entonación y cualquier información de carácter gestual y corporal.

Atención en el desarrollo de historias, cuentos motores e indicaciones de los adultos manifestando interés y participando en todas las actividades planificadas.

Coordinación, precisión en el movimiento en el momento de desarrollar diferentes actividades relacionadas con la expresión corporal.

Control del tono facial, respiración, desplazamientos y lateralidad. 
Coordinación dinámica óculo-manual, pédica y segmentaria.

\section{Conclusiones}

La conciencia del cuerpo, el conocimiento del espacio, la función tónica, la coordinación gruesa y fina, el equilibrio y la locomoción no son funciones aisladas y fragmentadas, sino que representan posibilidades de acción del niño y medios para conocer el mundo y relacionarse con los demás. En la acción corpórea, el niño se acerca a su realidad y es capaz de representar esa realidad en todas las esferas de sus procesos cognitivos.

Las actividades psicomotrices del niño favorecen la construcción de nociones espaciales, temporales y de integración del esquema corporal; a través de su cuerpo se relaciona con los objetos, las personas y el mundo social circundante, esto ayuda en la adquisición del aprendizaje y desarrolla sus capacidades, destrezas que se pueden direccionar de forma metódica a las esferas del lenguaje y la comunicación.

Las actividades lúdicas, el juego y otras acciones didácticas deben ser planificados cuando se busca de forma intencional aportar al aprendizaje, estas deben propiciar una experiencia vivida y gratificante en su naturalidad y cotidianidad.

La conciencia del cuerpo, el conocimiento del espacio, la función tónica, la coordinación gruesa y fina, el equilibrio y la locomoción no son funciones aisladas y fragmentadas, sino que representan posibilidades de acción para el niño y medios para conocer el mundo desde la relación con los demás. En la acción corpórea, el niño se acerca a su realidad y mediante la acción psicomotora primero gruesa, y luego fina es capaz de representar esa realidad en su expresión oral y escrita.

\section{Bibliografía}

1. De Sousa, B. S. Descolonizar el saber, reinventar el poder. Montevideo - Uruguay: Ediciones Trilce.. Extensión Universitaria de la República. 2010.

2. Doidge, N. El cerebro se cambia a sí mismo Ediciones Generales. España: Santillana. Ediciones Generales, S.L. 2008.
3. Escribano, G. A. Aprender a enseñar. Fundamentos de didáctica general. Cuenca: Humanidades. $2^{\circ}$ edición corregida y aumentada. Universidad de Catilla - La Mancha. 2004.

4. Fernández, M. Propuesta de Aprendizaje de la Lengua Escrita. México, D.F. 2005.

5. Gallego, R. S. Desarrollo General del Niño: El niño va creciendo. Innovación y Experiencias Educativas. 2010.

6. Gallo, C. L. Las prácticas corporales en la educación corporal. Ciencias - deporte, Florianópolis, V.34 - N4 Universidad de Antioquia, Grupo de Investigación: Estudios en Educación, 2012. 825 - 843.

7. González, G. M. La escritura en primer año de la escuela primaria y la Psicomotricidad . Psicomotricidad, Movimiento y Emoción, 2015. Vol. 1, No. 1.

8. Justo, M. E. Desarrollo Piscomotor en Educación Infantil. Almeira - España: Universidad del Almeira. 2014.

9. Justo, M. e. Desarrollo Psicmotor en Educación Infantil. Baes para la intervención. Almeria - España: Universidad de Almería. 2014

10. Larrea, d. G. El Currículo de la Educación Superior desde la Complejidad Sistémica. algunas consideraciones para orientar el proceso de construcción del nuevo modelo de formación universitaria. . Quito: CES. Consejo Educación Superior. 2014

11. León, L. C. Secuencias de desarrollo infantil integral. . Caracas, Venezuela: Universidad Católica Andrés Bello. 2008.

12. Núñez, S., \& Fernández, V. La Educación Psicomotriz. Lima / Perú: Eximpress S.A. 2010.

13. Oseda, G. J., Mendivel, J. R., \& Zevallos, S. L. Psicomotricidad e iniciación a la escritura en niños de 5 años. Apuntes de Ciencia \& Sociedad. Universidad Continental. 2015 Vol.5 No 1, 59 - 64. ISSN: 222 5- $515 \mathrm{X}$. 
14. Owens, R. El desarrollo del Lenguaje. . Madrid, España: PEARSON. Prentice Hall. 2008

15. Piaget, J. Psicología del niño. Barcelona España. Nueva edición por editorial 2002: Edit Morata. .

16. PNBV, S. N. (2013 - 2017). Plan Nacional del Buen Vivir 2013 - 2017. Todo el mundo mejor. Quito: SENPLADES. Primera edicion. ISBN-978-9942-07-448-5. www.buenvivir.gob.ec.

17. SENESCYT. Secretaría de Educación Superior, Ciencia, Tecnología e Innnovación. Sistema de Nivelación y
Admisión. Proyecto Integrador de Saberes. Proceso metodológico. Quito: Senescyt. Recuperado el 27 de Febrero de 2016, de educacionsuperior.gob.ec: http://prometeo.educacionsuperior.gob.ec/

18. Vargas, R. J., \& Arán, F. Importancia de la Parentalidad para el Desarrollo Cognitivo Infantil: una Revisión Teórica. Revista Latinoamericana de Ciencias Sociales, Niñez y Juventud, 2014 171-186.

19. Yturralde, T. E. La Lúdica.org. 2014 Obtenido de http://www.ludica.org/ 